УДК 33.013 .6

\title{
СОЗДАНИЕ РАСЧЕТНОЙ МОДЕЛИ ПРОЦЕССОВ СЖИГАНИЯ ТВЕРДЫХ БЫТОВЫХ ОТХОДОВ
}

\section{Магера Ю.М.}

\section{ПАО «Киевэнерго», пл. И. Франко, 5, Киев, 01001, Украина}

Створено розрахункову модель процесу спалювання твердих побутових відходів, що дозволяє вирішувати питання щодо необхідності спалювання додаткового палива у кожному конкретному випадку та визначати його необхідну витрату.
Создана расчетная модель процесса сжигания твердых бытовых от- combustion process calculation that ходов, позволяющая решать вопрос allows solving a problem whether о необходимости сжигания дополни- burning of additional fuel in certain тельного топлива в каждом конкрет- particular case is necessary and ном случае и определять его требуе- determining its required flow rate is мый расход. created.

Библ. 8, рис. 3.

Ключевые слова: твердые бытовые отходы, сжигание в плотном слое, температурный уровень топки, стабилизация температурного уровня, механический недожог.

В мире довольно распространена технология сжигания твердых бытовых отходов (ТБО). Так число мусоросжигающих заводов существенно превышает две тысячи. При этом доминируют процессы сжигания ТБО в плотном слое на механизированных решетках, хотя в достаточной мере отработаны и другие варианты, например, сжигание ТБО в кипящем слое [1-3].

На постсоветском пространстве имеется всего лишь несколько заводов по сжиганию ТБО. В Украине это завод «Энергия» в г. Киеве, в РФ это два мусоросжигающих завода в г. Москве и один завод в г. Череповце [1].

При таком значительном количестве мусоросжигающих заводов за рубежом, очевидно, что практические аспекты их эксплуатации отработаны достаточно хорошо. Имеется ряд компаний, которые готовы провести полный комплекс работ по созданию таких предприятий: от проектирования до отладки оборудования. Что касается Украины, то, с одной стороны, в виду малого количества таких предприятий, невозможно говорить о наличии достаточного отечественного опыта, однако, с другой стороны есть научно-производственные компании, которые имеют некоторый опыт проектирования и наладки предприятий по сжиганию ТБО [3].

При этом аналитический обзор по вопросам сжигания ТБО показал, что научное сопровождение этих технологий нуждается в дальнейшем совершенствовании. Так, например, в информационных источниках не имеется сведений о наличии адаптированных общепризнанных математических моделей, как гносеологического плана, так и упрощенных прогнозных моделей.

Одной из основных причин этой ситуации является то, что процессы сжигания ТБО необычайно сложны. Морфологический и гранулометрический составы исходного топлива настолько разнообразны, что практически невозможно выбрать представительный размер частицы ТБО, трансформации которой в топочном пространстве можно было бы отследить [1].

С другой стороны, вполне вероятно, что разработки математических моделей произведены научно-производственными компаниями, специализирующимися в данной области, и используются ими при проектировании и наладке заводов по сжиганию ТБО, однако сознательно не освещаются в открытой печати в коммерческих целях.

В условиях Украины на реализацию технологии сжигания ТБО накладывается ряд существенных особенностей, связанных с низкой и нестабильной теплотой сгорания ТБО [4]. При этом вполне вероятно, что иностранные разработки не будут в полной мере удовлетворять 
украинским реалиям. Для распространения этой технологии необходимы собственные теоретические и конструкторские разработки. Так в плане моделирования необходимы разработки для поддержки проектирования устройств по сжиганию ТБО. Отдельный класс моделей необходим для наладки и возможно в перспективе для работы в качестве компонента прогнозного управления или системы диагностики.

Одной из важнейших задач при сжигании ТБО является поддержание заданной температуры горения в топке, так как при низких темпеpaтурах (ниже $830 \ldots 850{ }^{\circ} \mathrm{C}$ ) может иметь место незавершенность химических процессов в газовой фазе, а при высоких температурах (более $850 \ldots 900{ }^{\circ} \mathrm{C}$ ) начинается зашлаковывание теплообменных поверхностей котла-утилизатора.

При изменении теплоты сгорания исходного ТБО может появляться необходимость в сжигании дополнительного высококалорийного топлива (обычно, природного газа). Текущие значения расхода дополнительного топлива могут быть установлены по ходу работы при помощи специальных регуляторов. Однако на стадии проектирования важно оценить ожидаемые максимальные расходы дополнительного топлива для заданной производительности топки по сжиганию ТБО и наиболее низкой теплоты сгорания ТБО. Кроме того, на стадии проектирования целесообразно выбрать рациональные значения коэффициента расхода воздуха, дать как можно более точные оценки производительностей вентилятора и дымососа.

Не менее важной задачей является создание моделей для прогнозного управления и систем диагностики, которые могли бы, работая в рамках системы АСУ ТП, параллельно с основными контурами регулирования выдавать прогноз поведения оборудования (особенно в нештатных ситуациях) и давать рекомендации по выбору рациональных технологических параметров дальнейшей работы.

Очевидно, что на первом этапе разрабатываемые модели будут основаны не на решении определенного набора дифференциальных уравнений (что, по мнению ряда исследователей, является необходимым признаком детерминированной математической модели [5]), а будут представлены набором расчетных зависимостей, отражающих интегральные материальные и энергетические балансы для отдельных этапов процесса, и отработанным механизмом обмена данными между этими расчетными блоками [6]. В данной работе предложен вариант такой математической модели (рис. 1).

Ее структура представлена шестью взаимодействующими расчетными блоками, два из которых (Блоки №1 и №2) являются вспомогательными. С их помощью можно находить дополнительные резервы для оптимизации процесса сжигания. Содержание блоков №1 и №2 отработано на концептуальном блоке и завершилось разработкой шаблонов расчетных выражений. Для установления конкретного вида уравнений связи необходимо проведение соответствующих экспериментальных исследований и обработка их результатов. Что касается основных расчетных блоков №3 - №6 они в полной мере отработаны и протестированы для условий предприятия завод «Энергия». Ниже представлено описание всех блоков математической модели.

Блок 1. Материальный баланс предварительного бункера с учетом стока части жидкости

В качестве исходных данных задается рабочий состав ТБО, поступающего на предприятие, \%: $\mathrm{C}_{\text {исх }}^{\mathrm{p}}, \mathrm{H}_{\text {исх }}^{\mathrm{p}}, \mathrm{N}_{\text {исх }}^{\mathrm{p}}, \mathrm{O}_{\text {исх }}^{\mathrm{p}}, \mathrm{S}_{\text {исх }}^{\mathrm{p}}, \mathrm{A}_{\text {исх }}^{\mathrm{p}}, \mathrm{W}_{\text {исх }}^{\mathrm{p}}$.

Для определения уменьшения влажности ТБО за счет стока части жидкости предложено использование эмпирической зависимости вида:

$$
\Delta \mathrm{W}=\mathrm{f}\left(\mathrm{H}, \tau, \mathrm{W}_{\text {исх }}^{\mathrm{p}}\right),
$$

где Н - высота слоя ТБО в предварительном бункере, м;

$\tau$ - среднее время нахождения конкретной порции ТБО в предварительном бункере (от входа до отгрузки в топочное пространство), ч.

Сам вид зависимости легко может быть установлен путем обработки данных о количестве жидкости, отводимой из промежуточного бункеpa, расходе ТБО и высоте слоя ТБО. Ввиду того, что данный элемент является подобным практически во всех случаях сжигания ТБО, полученный результат будет носить достаточно универсальный характер. Предположительно искомая зависимость имеет экспоненциальный вид.

После стока жидкости массовый расход ис- 
ходного ТБО $\left(\mathrm{G}_{\text {исх }}\right.$, кг/ч), перед поступлением в топку уменьшается на $\mathrm{G}_{\text {исх }} \cdot \Delta \mathrm{W} / 100$.

Состав ТБО, поступающего в топку, определяется при помощи коэффициента пересчета $\mathrm{k}=\frac{100}{100-\Delta \mathrm{W}}$ и составляет: $\mathrm{C}_{\mathrm{T}}^{\mathrm{p}}=\mathrm{k} \cdot \mathrm{C}_{\text {исх }}^{\mathrm{p}}$;

$\mathrm{H}_{\mathrm{T}}^{\mathrm{p}}=\mathrm{k} \cdot \mathrm{H}_{\text {исх }}^{\mathrm{p}} ; \mathrm{O}_{\mathrm{T}}^{\mathrm{p}}=\mathrm{k} \cdot \mathrm{O}_{\text {исх }}^{\mathrm{p}} ; \mathrm{N}_{\mathrm{T}}^{\mathrm{p}}=\mathrm{k} \cdot \mathrm{N}_{\text {исх }}^{\mathrm{p}} ;$

$\mathrm{S}_{\mathrm{T}}^{\mathrm{p}}=\mathrm{k} \cdot \mathrm{S}_{\text {исх }}^{\mathrm{p}} ; \mathrm{A}_{\mathrm{T}}^{\mathrm{p}}=\mathrm{k} \cdot \mathrm{A}_{\text {исх }}^{\mathrm{p}} ; \mathrm{W}_{\mathrm{T}}^{\mathrm{p}}=\mathrm{k} \cdot\left(\mathrm{W}_{\text {исх }}^{\mathrm{p}}-\Delta \mathrm{W}\right)$.
Понижение влажности ТБО, поступающего в топку, является важным положительным результатом. С одной стороны, конкретный уровень понижения влажности необходимо учитывать при проведении расчетов, с другой, стороны, необходим поиск технологических и конструктивных параметров, позволяющих добиваться как можно большего понижения влажности ТБО.

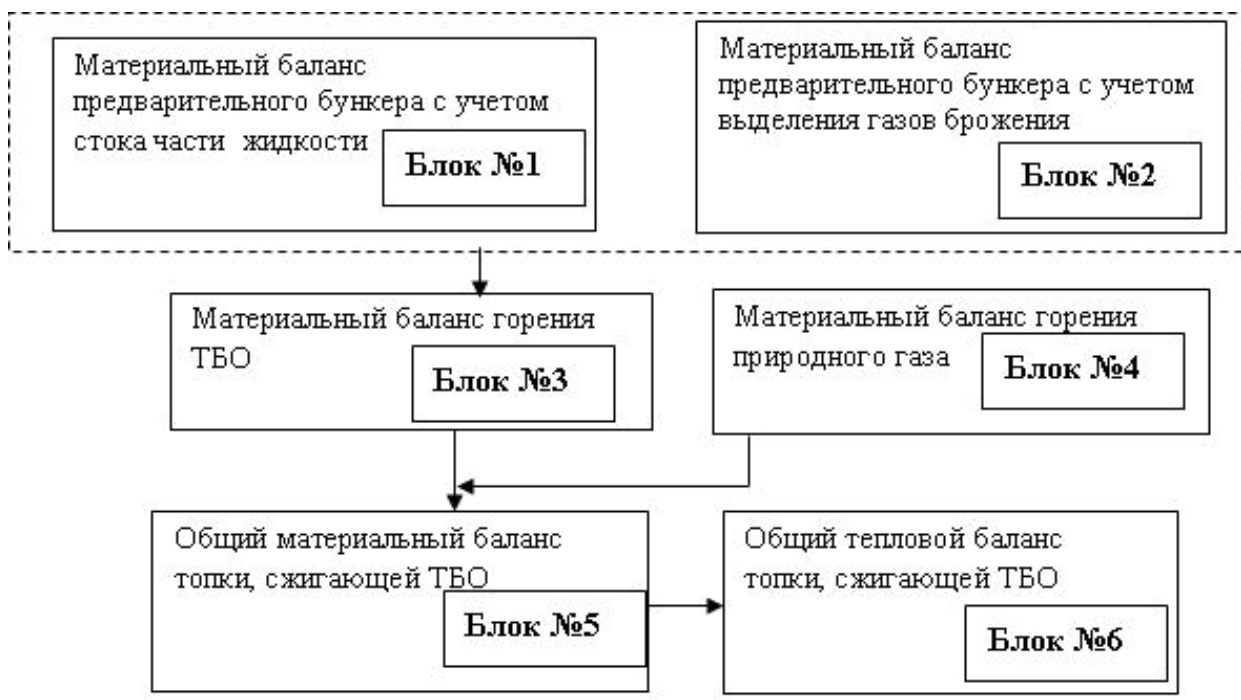

Рис. 1. Схема элементов упроценной математической модели процессов сәсгания ТБО.

Блок 2. Материальный баланс предварительного бункера с учетом выделения газов брожения

Отвод горючих газов брожения из предварительного бункера в топочное пространство и их сжигание существенно стабилизируют процессы горения ТБО. Оценку количества выделяющихся газов можно произвести при помощи эмпирических зависимостей. Одним из важнейших компонентов газов брожения является метан. Удельный выход метана, по мнению авторов, ( $\left.{ }^{3} / \kappa г\right)$ может быть описан при помощи эмпирической зависимости, типа:

$$
\mathrm{V}_{\text {сн4 }}=\mathrm{f}\left(\mathrm{H}_{\text {исх }}^{\mathrm{p}}, \mathrm{t}, \tau\right),
$$

где $\mathrm{H}_{\text {исх }}$ - содержание водорода в исходном ТБО, поступающем в предварительный бункер, \%; $\mathrm{t}$ - температура, при которой ТБО находится в предварительном бункере, ${ }^{\circ} \mathrm{C}$;

$\tau-$ среднее время нахождения конкретной порции ТБО в предварительном бункере (от входа до отгрузки в топочное пространство), ч.

Для получения этих уравнений понадобится обработка экспериментального материала, связанного с составом газов брожения, отводимых из предварительного бункера.

Аналогичные эмпирические уравнения связи могут быть получены и для других компонентов газов брожения. Общее количество газов брожения $\left(\mathrm{V}_{\text {г.бр }}\right)$ может быть получено путем суммирования удельного выхода всех компонентов. Тогда общее количество образующихся газов составляет $\mathrm{V}_{\text {г.бр }} \cdot \mathrm{G}_{\text {исх }}$.

При помощи достаточно простых зависимостей имеется возможность перейти к уменьшению массы ТБО, поступающего в топку. Хотя оно, как правило, не является значительным, а интерес представляет именно определение расхода и состава газов брожения. Эти газы через специальную систему каналов поступают в топочное пространство и сгорают в газовом пространстве топки над слоем горящего ТБО, что в некоторой 
мере стабилизирует условия горения.

\section{Блок 3. Материальный баланс горения ТБО}

Эта процедура выполняется с использованием зависимостей, применяемых для расчетов горения твердого топлива [7]. В ряде случаев зависимости используются в стандартном виде, а для учета эффектов, связанных с недожогом ТБО, они несколько видоизменяются.

При выполнении расчетов последовательно определяются теоретически необходимое для горения 1 кг ТБО количество кислорода; теоретически необходимое для горения 1 кг ТБО количество влажного воздуха; действительное количество влажного воздуха для горения 1 кг ТБО; выход продуктов сгорания при сжигании 1кг ТБО; общее количество продуктов сгорания от сжигания 1 кг ТБО; составляется материальный баланс процесса горения ТБО.

В случае необходимости учета механического недожога в данной работе предлагается следующий подход. Недовыделение химической энергии связано с тем, что в шлаках и золе остается некоторое количество несгоревшего углерода $\Delta \mathrm{C}_{\text {нед }}$. Эта величина определяется в зависимости от заданного уровня механического недожога $\left(\mathrm{q}_{4}, \%\right)$ по следующей зависимости:

$$
\Delta \mathrm{C}_{\text {нед }}=\frac{\mathrm{Q}_{\text {нр }}^{\mathrm{TБO}} \cdot \mathrm{q}_{4}}{33400},
$$

где $\mathrm{Q}_{\mathrm{Hр}}^{\mathrm{TБO}}$ - теплота сгорания ТБО, рассчитанная для его рабочего состава, кДж/кг; 33400 - теплота сгорания углерода, кДж/кг.

При этом количество углекислоты, образующейся при сжигании 1 кг ТБО, определяется не по стандартной зависимости: $\mathrm{V}_{\mathrm{CO}_{2}}^{\mathrm{TbO}}=0.0187 \cdot C_{\mathrm{T}}^{\mathrm{p}}$, a как $\mathrm{V}_{\mathrm{CO}_{2}}^{\mathrm{TbO}}=0.0187 \cdot\left(C_{\mathrm{T}}^{\mathrm{p}}-\Delta C_{\text {нед }}\right)$, а выражение для определения количества кислорода в продуктах сгорания 1 кг ТБО записывается как:

$$
V_{\mathrm{O}_{2}}=k_{\mathrm{O}_{2}} \cdot(\alpha-1) \cdot \frac{\mathrm{V}_{\mathrm{O}_{2} \text { теор }}}{\mathrm{k}_{\mathrm{O}_{2}}}+0.0187 \cdot \Delta C_{\text {нед }},
$$

где $\alpha$ - коэффициент расхода воздуха;

$\mathrm{V}_{\text {О2теор }}$ - теоретически необходимое для горения 1 кг ТБО количество кислорода, м $^{3} /$ кг.

Важным элементом этого блока является проверка схождения материального баланса. При этом сравниваются масса веществ, вступивших в

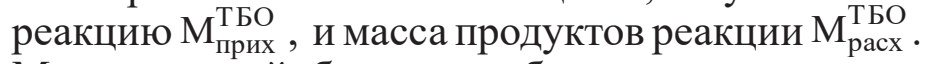
Материальный баланс удобно строить относи- тельно 1 кг ТБО. Тогда:

$$
\begin{gathered}
\mathrm{M}_{\text {Прих }}^{\text {ТБО }}=1+\mathrm{V}_{\text {вл. в.дейст }}^{\text {ТБО }} \rho_{\text {вл.в }} ; \\
\mathrm{M}_{\mathrm{pacx}}^{\mathrm{TБО}}=\mathrm{V}_{\text {пс }}^{\text {ТБО }} \cdot \rho_{\text {пс }}^{\text {ТБО }}+\mathrm{A}_{\mathrm{T}}^{\mathrm{p}} / 100+\Delta \mathrm{C}_{\text {нед }} / 100 .
\end{gathered}
$$

Плотности влажного воздуха ( $\left.\rho_{\text {вл.в }}\right)$ и продук-

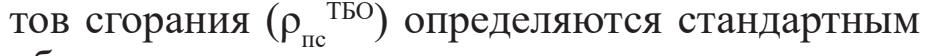
образом в зависимости от их состава как для газовой смеси:

$$
\rho_{\mathrm{cM}}=\frac{\sum_{\mathrm{i}=1}^{\mathrm{n}} \mathrm{C}_{\mathrm{i}} \cdot \mathrm{M}_{\mathrm{i}}}{22,4 \cdot 100},
$$

где $\mathrm{C}_{\mathrm{i}}$ - содержание i-го компонента, \%; $\mathrm{M}_{\mathrm{i}}$ - молярная масса і-го компонента, кг/кмоль; $\mathrm{N}$ - количество компонентов смеси.

\section{Блок 4. Составление материального баланса горения природного газа}

Для реализации этого блока используется стандартная методика, применяемая для расчета горения газовых смесей. При этом последовательно определяются теоретически необходимое для горения $1 \mathrm{~m}^{3}$ природного газа количество кислорода; теоретически необходимое для горения $1 \mathrm{M}^{3}$ природного газа количество влажного воздуха; действительное количество влажного воздуха для горения $1 \mathrm{~m}^{3}$ природного газа; выход продуктов сгорания при сжигании $1 \mathrm{~m}^{3}$ природного газа; составляется материальный баланс процесса горения природного газа.

Блок 5. Составление общего материального баланса топки при совместном сжигании ТБО и

\section{природного газа}

Эта процедура выполняется с использованием результатов, полученных в Блоках №3 и №4. Для случая совместного сжигания вводится понятие доли природного газа $\xi$, которая показывает, сколько добавленных кубических метров природного газа соответствует 1кг ТБО.

Объемы компонентов продуктов сгорания определяются путем суммирования выходов компонентов при горении ТБО с выходами соответствующих компонентов при горении природного газа, умноженными на $\xi$. Например,

$$
\mathrm{V}_{\mathrm{CO}_{2}}=V_{\mathrm{CO}_{2}}^{T Б O}+\xi \cdot V_{\mathrm{CO}_{2}}^{\Pi \Gamma} \text {. }
$$

Приходная и расходная статьи материального баланса могут быть определены при помощи следующих выражений: 


$$
\mathrm{M}_{\text {прих }}=1+\xi \cdot \rho_{\text {ПГ }}+\left(\mathrm{V}_{\text {вл.в.дейст }}^{\text {ТБО }}+\xi \cdot \mathrm{V}_{\text {вл.в.дейст }}^{\text {ПГ }}\right) \cdot \rho_{\text {в.вл }} ; \mathrm{M}_{\text {расх }}=\mathrm{V}_{\text {пс }} \cdot \rho_{\text {пс }}+\mathrm{A}_{\mathrm{T}}^{\mathrm{p}} / 100+\Delta \mathrm{C}_{\text {нед }} / 100 .
$$

\section{Блок 6. Составление общего теплового баланса топки, сжигающей ТБО}

Уравновешивание теплового баланса производится за счет определения калориметрической температуры горения ТБО или ТБО совместно с природным газом. При этом используются результаты построения общего материального баланса топки при совместном сжигании ТБО и природного газа:

$$
\mathrm{t}_{\text {кал }}=\frac{\mathrm{Q}_{\mathrm{mp}}^{\mathrm{TБО}} \cdot\left(1-q_{4} / 100\right)+\xi \cdot \mathrm{Q}_{\text {нр }}^{\Pi Г}}{0,01 \cdot\left(\% \mathrm{CO}_{2} \cdot c_{\mathrm{CO}_{2}}\left(t_{\text {кал }}\right)+\% \mathrm{H}_{2} \mathrm{O} \cdot c_{\mathrm{H}_{2} \mathrm{O}}\left(t_{\text {кал }}\right)+\% \mathrm{O}_{2} \cdot c_{\mathrm{O}_{2}}\left(t_{\text {кал }}\right)+\% N_{2} \cdot c_{\mathrm{N}_{2}}\left(t_{\text {кал }}\right)+\% \mathrm{SO}_{2} \cdot c_{\mathrm{SO}_{2}}\left(t_{\text {кал }}\right)\right)},
$$

где $\% \mathrm{CO}_{2}, \% \mathrm{H}_{2} \mathrm{O}, \% \mathrm{O}_{2}, \% \mathrm{~N}_{2}, \% \mathrm{SO}_{2}$ - процентное содержание соответствующих компонентов в продуктах сгорания, \%;

$\mathrm{c}_{\mathrm{CO} 2}(\mathrm{t}), \mathrm{c}_{\mathrm{H} 2 \mathrm{O}}(\mathrm{t}), \mathrm{c}_{\mathrm{O} 2}(\mathrm{t}), \mathrm{c}_{\mathrm{N} 2}(\mathrm{t}), \mathrm{c}_{\mathrm{SO} 2}(\mathrm{t})-$ теплоемкости соответствующих компонентов продуктов сгорания, вычисленные для заданного значения температуры.

Определение калориметрической температуры производится при помощи итерационной процедуры, поскольку искомая величина ткал входит в выражение для своего определения как аргумент для вычисления теплоемкостей компонентов продуктов сгорания. Расчетная процедура представлена следующим алгоритмом:

- предварительно задается значение $\mathrm{t}_{\text {кал }}$;

- вычисляются значения теплоемкостей газовых компонентов;

- при помощи выражения (1) определяется новое значение $\mathrm{t}_{\text {кал; }}$;

- производится сравнение предварительно заданного и определенного значений $\mathrm{t}_{\text {кал }}$ В случае совпадения этих значений в пределах заданной погрешности, расчет считается завершенным. При невыполнении этого условия определенное значение $\mathrm{t}_{\text {кал }}$ используется в качестве заданного на первом шаге данной процедуры.

Действительное значение температуры горения в топке может быть определено при помощи выражения:

$$
\mathrm{t}_{\text {д }}=\mathrm{k}_{\text {пир }} \cdot \mathrm{t}_{\text {кал }} \text {, }
$$

где $\mathrm{k}_{\text {пир }}$ - пирометрический коэффициент, учитывающий отличие действительного значения температуры от tкал по причине теплообменных процессов, протекающих в топке.

Значение $\mathrm{k}_{\text {пир }}$ является эмпирической величиной, которая должна отдельно определяться для каждого конкретного случая. Диапазон возможного изменения значений $\mathrm{k}_{\text {пир }}$ составляет $0,5 \ldots 0,9$. Для автоматизации расчетной процедуры по определению $\mathrm{t}_{\text {кал }}$ и $\mathrm{t}_{\text {д }}$ необходимо наличие функциональных зависимостей теплоемкостей компонентов продуктов сгорания от температуры. Для этой цели в современном инженерно-вычислительном пакете обработаны соответствующие табличные данные [8] и получены уравнения регрессии. Так, например, для углекислоты зависимость теплоемкости от температуры аппроксимирована полиномом второй степени и получено следующее уравнение регрессии:

$$
\begin{gathered}
\mathrm{c}_{\mathrm{CO} 2}(t)=1.661+6.388 \cdot 10^{-4} \cdot t-1.209 \cdot 10^{-8} \cdot t^{2}, \mathrm{\kappa} \text { Дж/(м3·К). } \\
\text { Схема использование разработанной } \\
\text { математической модели }
\end{gathered}
$$

Основное ядро модели представлено блоками №3-№6. Схема их использования представлена следующим алгоритмом:

1) выполняется расчет горения исключительного одного ТБО (Блок №3);

2) определяются калориметрическая и действительная температуры горения (Блок №6);

3) полученная действительная температура сравнивается с рекомендованным диапазоном $850 \ldots 900{ }^{\circ} \mathrm{C}$. В случае если она попадает в указанный диапазон, то в сжигании дополнительного топлива нет необходимости. Если она выше (что очень маловероятно) необходимо увеличить значение коэффициента расхода воздуха. Если $\mathrm{t}_{\text {д }}<850{ }^{\circ} \mathrm{C}$ необходимо сжигание дополнительного топлива;

4) выполняется расчет горения природного газа (Блок №4);

5) сводится общий материальный баланс со- 
вместного горения ТБО и природного газа с предварительно заданным значением доли природного газа $\xi$;

6) определяются калориметрическая и действительная температуры горения (Блок №6);

7) полученная действительная температура сравнивается с рекомендованным диапазоном $850 \ldots 900{ }^{\circ} \mathrm{C}$. В случае ее непопадания в этот диапазон происходит возврат на 5-ый шаг, где корректируется значение $\xi$. Совокупность шагов 5-7 повторяется до тех пор, пока действительная температура в топке не войдет в заданный диапазон.

\section{Адаптаиия модели.}

Для достижения адекватности описания изучаемого процесса сжигания ТБО при помощи разработанной модели в конкретных условиях необходимо провести процедуру ее адаптации. Для решения этой задачи предлагается использовать процедуру начальной подстройки. При этом приведение в соответствие результатов замера и расчета одного из важнейших параметров процесса сжигания ТБО (температуры в топочном пространстве) достигается за счет коррекции значения пирометрического коэффициента. Определенные значения $\mathrm{k}_{\text {пир }}$ в полной мере соответствуют только тому объекту и режимам эксплуатации, для которых они были получены. Так, для сжигания ТБО величина $\mathrm{k}_{\text {пир }}$ зависит от тепловой нагрузки котла-утилизатора и понижается с ее увеличением.

В качестве первой точки, для которой проведена адаптация расчетной модели для условий котла-утилизатора, сжигающего ТБО на заводе «Энергия», были выбраны условия эксплуатации оборудования в номинальных условиях: состав ТБО, \%: $\mathrm{C}_{\mathrm{T}}{ }^{\Gamma}=56 ; \mathrm{H}_{\mathrm{T}}{ }^{\Gamma}=7,5 ; \mathrm{N}_{\mathrm{T}}{ }^{\Gamma}=1 ; \mathrm{S}_{\mathrm{T}}{ }^{\Gamma}=0,5$; $\mathrm{A}_{\mathrm{T}}{ }^{\mathrm{C}}=30 ; \mathrm{W}_{\mathrm{T}}^{\mathrm{P}}=30$; теплота сгорания ТБО 2500 ккал/кг; массовая производительность по сжигаемому ТБО - 15т/ч; объемный расход воздуха: первичный - 70 тыс. м³, вторичный 21 тыс. м $3 / 4$; уровень механического недожога $6 \%$; расход природного газа на подсветку отсутствует; температура подогретого воздуха, поступающего в топку $-180^{\circ} \mathrm{C}$; температура в топке $850{ }^{\circ} \mathrm{C}$

Согласно выполненным расчетам коэффициент расхода воздуха при таких расходных коэффициентах по топливу и окислителю составля- ет 2,12. При помощи программы, реализующей разработанный расчетный алгоритм произведен подбор значения $\mathrm{k}_{\text {пир }}$, при котором рассчитанное значение действительной температуры в топке составляло бы $850{ }^{\circ} \mathrm{C}$. Для приведенного выше набора исходных данных получено значение $\mathrm{k}_{\text {пир }}=0,735$.

Определение $\mathrm{k}_{\text {пир }}$ для других условий эксплуатации котла-утилизатора, сжигающего ТБО (меньшие теплоты сгорания ТБО и более низкие тепловые нагрузки котла) показали диапазон изменения $\mathrm{k}_{\text {пир }}$ от 0,735 до 0,9.

\section{Практические задачи, решаемые при помощуи разработанной модели}

Основной и очевидной задачей, решаемой при помощи разработанной модели, является решение вопроса о необходимости сжигания дополнительного топлива в каждом конкретном случае и определение его требуемого расхода. Также возможно решение задачи выбора рационального значения коэффициента расхода воздуха, предполагаемых максимальных производительностей вентилятора и дымососа, что требуется для подбора конкретного оборудования.

Ниже проиллюстрировано решение задачи определения удельного расхода природного газа на подсветку в зависимости от теплоты сгорания исходного ТБО для условий завода «Энергия» (г. Киев). Технологический процесс налажен таким образом, что при теплоте сгорания ТБО 1650 ккал/кг и более удается реализовать топочный процесс без расходования природного газа на подсветку при гарантированном поддержании температуры в топке на заданном уровне $850^{\circ} \mathrm{C}$. При дальнейшем понижении теплоты сгорания ТБО и работе с теми же значением коэффициента расхода воздуха появляется необходимость в дополнительном сжигании природного газа. Проведение вычислений для теплот сгорания от 1650 ккал/кг до 1250 ккал/кг при помощи разработанного расчетного комплекса позволило получить результаты, представленные на рис. 2.

Для дополнительного раскрытия возможностей расчетной модели проведена серия численных экспериментов по исследованию влияния величины коэффициента расхода воздуха на температуру в топке. В качестве исходной точки для расчетов выбран случай с теплотой сгорания 
ТБО 1650 ккал/кг с базовым значение коэффициента расхода воздуха 2.23. Далее расчеты производились для ряда точек с пониженным значением $\alpha$ в диапазоне от 2,23 до 1,7 (рис. 3). При этом для понимания степени влияния $\alpha$ на температурное состояние топки было снято ограничение по максимальному значению температуры в топке.
Анализ результатов показывает, что изменение коэффициента расхода воздуха в допустимых пределах (гарантирующих полное сжигание ТБО и максимально возможное подавление образования вредных соединений) является эффективным инструментом для управления температурным состоянием топки.

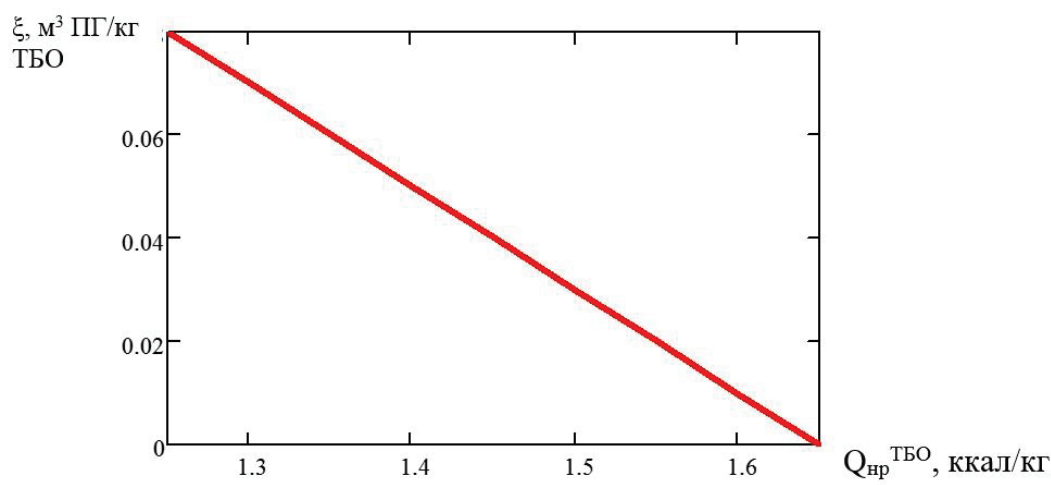

Рис. 2. Зависимость удельного расхода природного газа на подсветку от теплоты сгорания ТБО.

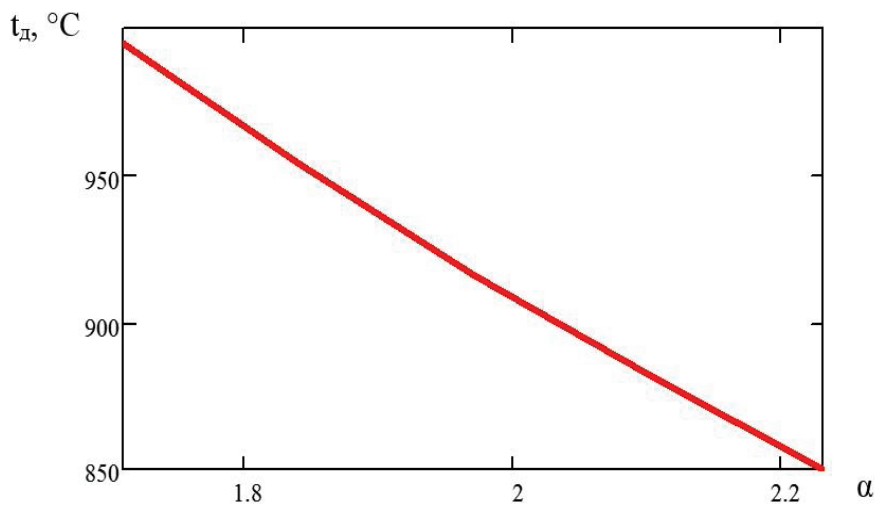

Рис. 3. Зависимость температуры в топке от значения коэффициента расхода воздуха для случая сжсигания ТБО с теплотой сгорания 1650 ккал/кг без использования природного газа для подсветки.

\section{Выводы}

Обоснована необходимость создания математических (расчетных) моделей сжигания ТБО для поддержки проектирования соответствующих предприятий, а также прогнозных моделей для повышения точности управления данным процессом и реализации его диагностики.

Создана расчетная модель процесса сжигания ТБО, основными элементами которой являются блоки по построению материальных балансов сжигания ТБО, природного газа, совместного сжигания ТБО и природного газа и теплового баланса топки, сжигающей ТБО. На концептуаль- ном уровне отработаны дополнительные блоки модели, связанные с построением материального баланса предварительного бункера с учетом стока части жидкости и материального баланса предварительного бункера с учетом выделения газов брожения.

Адаптация модели проведена для условий завода «Энергия» путем определения подстроечных значений пирометрического коэффициента для разных режимов эксплуатации. Для номинальных условий эксплуатации значение $\mathrm{k}_{\text {пир }}$ составляет 0,735. Определение $\mathrm{k}_{\text {пир }}$ для других условий эксплуатации котла-утилизатора, сжига- 
ющего ТБО (меньшие теплоты сгорания ТБО и более низкие тепловые нагрузки котла) показали диапазон изменения $\mathrm{k}_{\text {пир }}$ от 0,735 до 0,9.

Одной из основных задач, решаемых при помощи разработанной модели, является решение вопроса о необходимости сжигания дополнительного топлива в каждом конкретном случае и определение его требуемого расхода. Также возможно решение задачи выбора рационального значения коэффициента расхода воздуха, предполагаемых максимальных производительностей вентилятора и дымососа, что требуется для подбора конкретного оборудования.

В результате выполнения расчетов для условий завода «Энергия» установлены требуемые удельные расходы природного газа на подсветку для стабилизации температурного состояния в топке на уровне $850{ }^{\circ} \mathrm{C}$ для теплот сгорания ТБО ниже 1650 ккал/кг. Также расчетным путем обоснована высокая степень влияния коэффициента расхода воздуха на температурное состояние топки при прочих равных условиях.

\section{ЛИТЕРАТУРА}

1. Тугов А.Н. Исследование процессов и технологий энергетической утилизации бытовых отходов для разработки отечественной ТЭС на ТБО автореф. докт. дис. Москва, 2012.- 43 с.

2. Тугов А.Н. Использование энергопотенциала твердых бытовых отходов в России и за рубежом. А.Н. Тугов, Ф.В. Москвичев. Энергия: экономика, техника, экология. 2011. - №8. C. $32-42$.

3. Меллер В.Я. Пути использования ТБО. В.Я. Меллер. Проблемы экологии и эксплуатации объектов энергетики. Сборник Трудов.- Киев, 2016.- C. 200-204.

4. Магера Ю.М. Концепция развития завода «Энергия»: ТЭЦ на ТБО. Ю.М. Магера. Проблемы экологии и эксплуатации объектов энергетики. Сборник Трудов. - Киев, 2016. С. 208-211.

5. Ткаченко В.Н. Математическое моделирование, идентификация и управление технологическими процессами тепловой обработки материалов. В.Н. Ткаченко. Т.13. - Сер. «Задачи и методы: математика, механика, кибернетика». Киев: Наукова думка, 2008.- 244 с.

6. Кравизов B.B. Теплотехника термической переработки твердых топлив: Учебное пособие/ Кравцов В.В., Бирюков А.Б., Дробышевская И.П.Донецк: Издательство «Ноулидж», 2011. - 170 с.

7. Бирюков А.Б. Сжигание и термическая переработка органических топлив. А.Б. Бирюков, И.П. Дробышевская, Ю.Е. Рубан. - Донецк: Издательство «Ноулидж», 2014.- 230 с.

8. Казаниев Е.И. Промышленные печи: Справочное руководство для расчета и проектирования, 2-е издание дополненное и переработанное. Е.И. Казанцев.- М.: Металлургия, 1975. $-368 \mathrm{c}$. 


\section{CREATION OF MUNICIPAL SOLID WASTES COMBUSTION PROCESSES CALCULATION MODEL}

\section{Magera Y.M.}

JSC "Kyivenergo",

sq. I. Franko, 5, Kyiv, 01001, Ukraine

Calculation model for municipal solid wastes combustion process which main elements are blocks for drawing up municipal solid wastes combustion, natural gas combustion and joint combustion of these fuels material balances and thermal balance of the furnace is created. Adaptation of the model is conducted due to determining of pyrometric coefficient values for different exploitation regimes. For nominal exploitation conditions (for wastes combustion plant "Energiya") its values is estimated as 0.735 . One of the main problems solved by the model is technological challenge whether burning of additional fuel in certain particular case is necessary and determining of its required flow rate. It is also possible to solve a problem of rational values of air flow rate coefficient, maximal capacities of ventilator and exhauster estimation. As a results of calculation series required flow rates of natural gas for furnace heat state stabilization in case when municipal solid wastes heating value is lower then $1650 \mathrm{kcal} / \mathrm{kg}$ for conditions of wastes combustion plant "Energiya" are determined.

References 8, figures 3 .

Key words: municipal solid wastes, combustion in stagnant layer, furnace temperature level, temperature level stabilization, mechanical underburning.

1. Tugov A.N. Research of processes and technology of municipal solid wastes utilization for working out of domestic TPP using MSW, Avtoref. dokt. dis., Moskva. 2012. 43 p. (Rus.)

2. Tugov A.N. , Moskvichev A.N. Use of energy potential of municipal solid wastes in Russia and abroad . Energija: ekonomika, tehnika, ekologija. 2011. №8. P. 32-42. (Rus.)

3. Meller V.Ja. Ways of MSW use . Problemy ekologii i ekspluatacii objektov energetiki. Sbornik trudov. Kiev. 2016. P. 200-204. (Rus.)

4. Magera Ju.M. Concept of plant "Energija" development: TPP using MSW . Problemy ekologii i ekspluatacii objektov energetiki. Sbornik trudov. Kiev. 2016. P. 208-211. (Rus.)

5. Tkachenko V.N. Mathematical modeling, identification, and control of materials heat processing technological processes . V.13. Ser. «Zadachi i metody: matematika, mehanika, kibernetika».-Kiev: Naukova dumka, 2008. 244 p. (Rus.)

6.KravcovV.V., BirjukovA.B., DrobyshevskajaI.P. Heat engineering of solid fuels thermal processing: Uchebnoe posobie., Doneck: Izdatelstvo «Noulidzh», 2011. 170 p. (Rus.)

7.BirjukovA.B., DrobyshevskajaI.P., RubanJu.E. Combustion and thermal processing of organic fuels. Doneck: Izdatelstvo «Noulidzh». 2014. 230 p. (Rus.)

8. Kazancev E.I. Industrial furnaces: Reference for calculation and design, 2-e izdanie dopolnennoe i pererabotannoe, M.: Metallurgija, 1975. 368 p. (Rus.)

Получено 08.11.2016 Received 08.11.2016 